\title{
Splenic and Portal Vein Thrombosis With Concurrent Splenic Infarction: A Rare Manifestation of Essential Thrombocythemia
}

\author{
Manisha Gulia ${ }^{1}$, Neha Sharma ${ }^{1}$, Yajur Arya ${ }^{1}$, Arshi Syal ${ }^{1}$, Monica Gupta ${ }^{1}$ \\ 1. Internal Medicine, Government Medical College and Hospital, Chandigarh, IND
}

Corresponding author: Monica Gupta, monicamanish2001@gmail.com

\begin{abstract}
Mesenteric venous thrombosis (MVT) presents with a wide range of clinical presentations depending on the vessel involved, degree of thrombosis, and the extent of bowel wall ischemia. MVT usually has an insidious presentation and is often a forerunner of an underlying disorder. Essential thrombocythemia (ET) presenting itself as MVT along with splenic infarction is a rare presentation. Here, we report the case of a 54-year-old female with massive splenomegaly, thrombocytosis, and acute splenic and portal venous thrombosis along with multiple splenic infarcts. Bone marrow suggested ET with JAK2V617F mutation positivity. She was managed conservatively and made an uneventful recovery.
\end{abstract}

Review began 05/27/2021 Review ended 06/08/2021 Published 06/20/2021

(๑) Copyright 2021

Gulia et al. This is an open access article distributed under the terms of the Creative Commons Attribution License CC-BY 4.0., which permits unrestricted use, distribution, and reproduction in any medium, provided the original author and source are credited.
Categories: Internal Medicine, Oncology, Hematology

Keywords: essential thrombocythemia, mesenteric venous thrombosis, splenic infarction, portal vein thrombosis, myeloproliferative disorder

\section{Introduction}

Essential thrombocythemia (ET) is an acquired myeloproliferative disorder (MPD) characterized by a persistent elevation of platelet count. The annual incidence has been estimated between 0.77 and 2.53 per 100,000 people and is comparably more common in young females [1]. The clinical manifestations range from vaso-occlusive events to bleeding manifestations and transformation into leukemia or other myeloid neoplasms at later stages. Thrombosis at unusual sites is rare and happens to be a severe complication of ET [1]. Around $8-15 \%$ of the cases are idiopathic and the remaining have a direct relation with various conditions, particularly MPD [2]. Early diagnosis at these unusual sites is crucial in patients with ET as thrombosis significantly affects the disease outcome and is associated with severe organ damage and high mortality [3].

\section{Case Presentation}

A 54-year-old female, known case of diabetes mellitus, hypertension, coronary artery disease, and seizure disorder presented with a complaint of pain in the left upper abdomen for the preceding five days. The pain was intermittent, moderate in intensity along with radiation to the back. There was no association of abdominal pain with eating. It was not associated with nausea, vomiting, or diarrhea. On examination, the patient was conscious, oriented, afebrile and her vitals were in the normal range. On abdominal examination, there was tenderness in the left hypochondrium, and the spleen was palpable four centimeters (cm) below the costal margin. Bowel sounds were normal and there were no signs of peritoneal inflammation. Cardiovascular, respiratory, and neurological examinations were unremarkable.

Investigations revealed thrombocytosis $\left(1101 \times 10^{9} / \mathrm{L}\right)$, leukocytosis $\left(33.7 \times 10^{9} / \mathrm{L}\right)$, hemoconcentration with hemoglobin of $12.5 \mathrm{~g} / \mathrm{dL}$, and a hematocrit of $48 \%$. Peripheral blood film examination revealed anisopoikilocytosis, moderate hypochromia, leukocytosis, and thrombocytosis. There was no peripheral evidence of myelofibrosis. Renal and liver function tests were normal. Ultrasound abdomen was suggestive of splenomegaly $(17.7 \times 7 \mathrm{~cm})$ along with splenic abscesses and portal vein thrombosis. The findings were confirmed on a contrast-enhanced CT (CECT) scan of the abdomen which demonstrated hepatomegaly with acute splenic and portal venous thrombosis with massive splenomegaly with areas indicating infarcts (Figure 1). 


\section{Cureus}

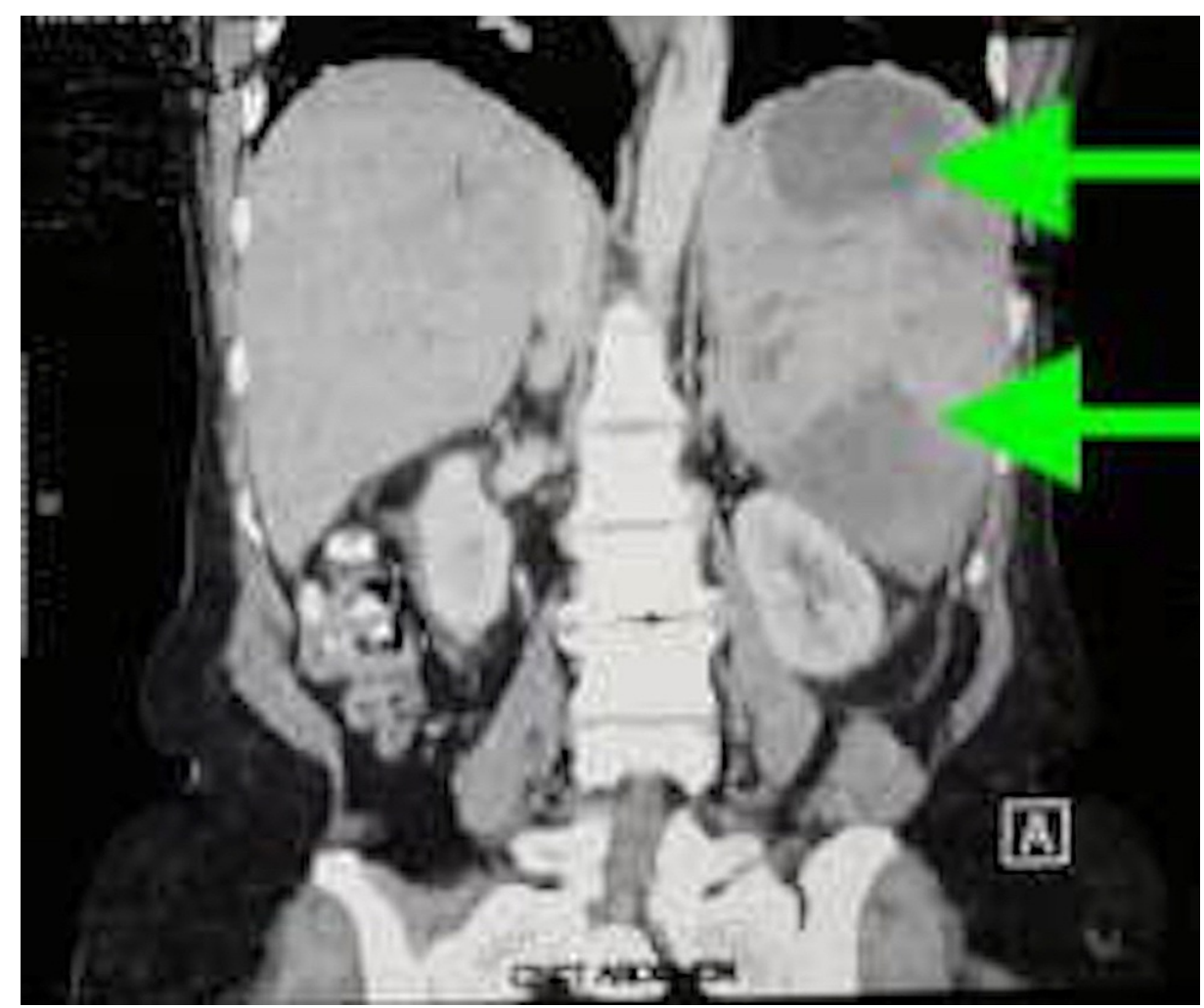

FIGURE 1: CECT of the abdomen showing hepatosplenomegaly with few irregular hypodense areas in the spleen suggestive of infarction (green arrows).

CECT: Contrast-enhanced CT.

Upper GI endoscopy revealed grade one esophageal varices. The stomach and duodenum appeared normal. Viral markers, antinuclear antibody panel, and tumor markers including cancer antigen 19-9 (CA 19-9), carcinoembryonic antigen (CEA), and alpha-fetoprotein (AFP) were negative. Transient elastography of the liver (FibroScan) was not suggestive of cirrhosis. Two-dimensional (2D) echocardiography revealed a normal left ventricular systolic function and no regional wall motion abnormalities or evidence of any clot or vegetation. Bone marrow examination revealed hypercellular marrow (80-85\% cellularity), megakaryocytic hyperplasia with focal clustering, and the presence of staghorn nuclei. These changes were suggestive of a myeloproliferative neoplasm, possibly ET (Figure 2). 


\section{Cureus}

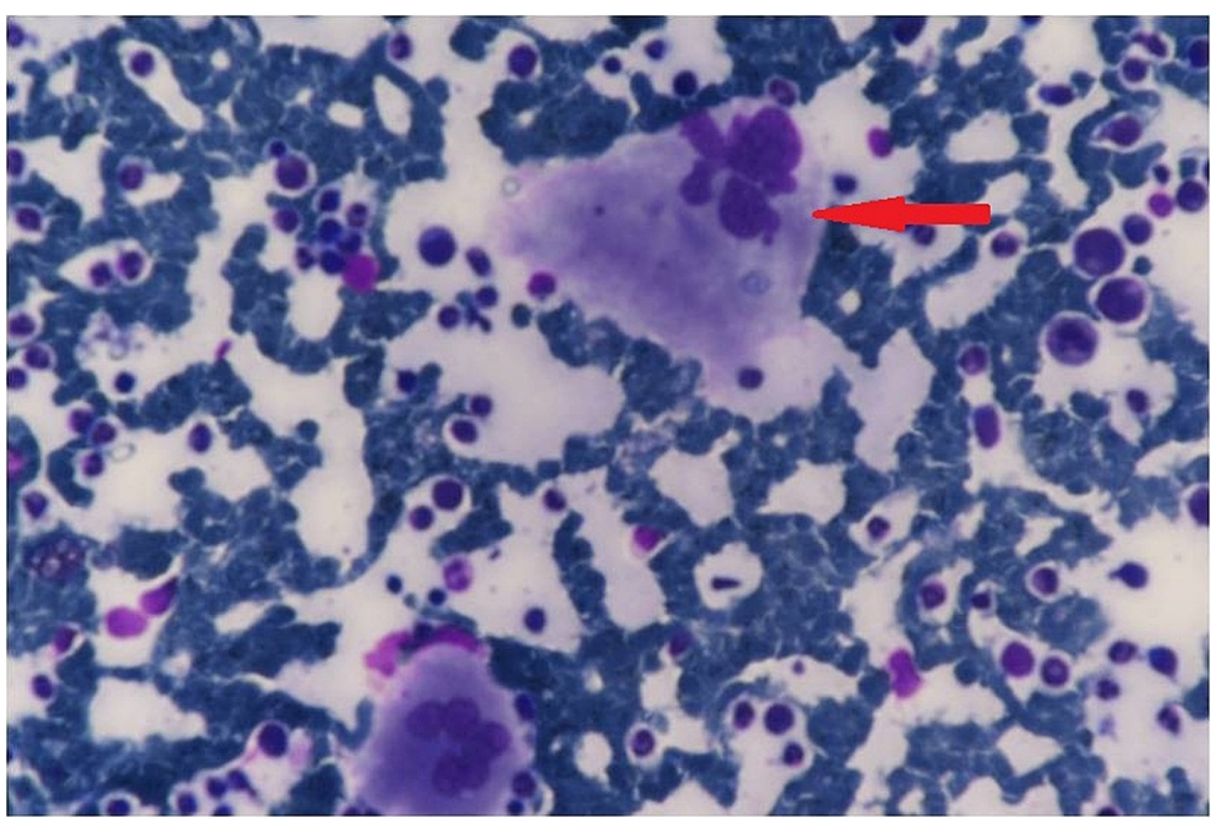

FIGURE 2: Bone marrow examination revealing a hypercellular marrow and megakaryocytic hyperplasia with staghorn nuclei (red arrow).

A molecular study revealed JAK2V617F mutation positivity and was negative for BCR-ABL, thrombopoietin receptor gene (myeloproliferative leukemia, MPL), and calreticulin (CALR gene) mutations.

A diagnosis of ET (high-risk disease) was made and the patient was started on hydroxyurea. Portal vein thrombosis was managed with heparin followed by warfarin with international normalized ratio (INR) monitoring daily while the patient was admitted and every three to five days after discharge. INR was maintained between two and three. On follow-up evaluation six weeks later, she was asymptomatic with a platelet count of $95 \times 10^{9} / \mathrm{L}$ and a total leucocyte count of $4.5 \times 10^{9} / \mathrm{L}$.

\section{Discussion}

Mesenteric venous thrombosis (MVT) involving mesenteric, portal, and splenic veins is a common pathology with an array of clinical manifestations. Risk factors include inflammatory diseases such as pancreatitis and inflammatory bowel disease, postoperative states, cirrhosis, antiphospholipid syndrome, paroxysmal nocturnal hemoglobinuria, malignancy, oral contraceptives, pregnancy, and the postpartum period $[4,5]$. Hematological conditions involving thrombophilia should also be kept under consideration in the evaluation of thrombotic episodes, such as prothrombin gene mutation and factor $\mathrm{V}$ Leiden gain-of-function mutations or hematopoietic system-related clonal disorders like MPD which include chronic myeloid leukemia (CML) and BCR-ABL negative disorders (polycythemia vera [PV], ET, and primary myelofibrosis [PMF]). BCR-ABL negative MPD is associated with a somatic mutation in JAK2 which has been identified in almost all cases of PV (>95\% positive), in $50-70 \%$ of ET and $50-60 \%$ of PMF patients [6]. Therefore, JAK2 being a pathognomonic feature of chronic MPD cannot be used for identifying the subtype, consequently, bone marrow examination remains mandatory in the diagnostic evaluation. In ET, bone marrow is normocellular or minimally hypercellular with megakaryocytosis which appears mature, multilobulated, either dispersed throughout the section or in loose clusters. This is in contrast to tightly packed, bizarre megakaryocytes seen in PMF with reticulin and collagen fibrosis [7,8]. Elevated inflammatory markers, such as ferritin, C-reactive protein (CRP), and erythrocyte sedimentation rate (ESR) with normal lactate dehydrogenase levels are seen in case of secondary thrombocytosis; however, these markers cannot be considered discriminatory at all as they can also be seen in ET.

ET clinically presents with vascular occlusive events and hemorrhages with transformation to leukemia or other myeloid diseases as a late complication. ET presenting with abdominal vein thrombosis is rare. In a study involving 460 patients with ET, Gangat et al. reported a prevalence rate of $4 \%$ for abdominal vein thrombosis [9]. In an acute variant, thrombocytosis and hyperviscosity lead to an increased thrombotic tendency. However, at later stages, functional alteration in platelet activity usually dominates.

ET usually has a good prognosis with regards to median survival. MVT is a poor prognostic marker due to leukemic or fibrotic transformation and hepatic failure [9]. Since there is no curative or disease-modifying therapy available currently, treatment strategies for ET include antiplatelet agents and cytoreductive therapy such as hydroxyurea, busulfan, anagrelide, and pegylated interferon-alfa. MPD-related variceal 
bleed is managed in the same way as due to cirrhosis or cancer, but the prognosis is comparatively better in the former case [10].

\section{Conclusions}

Our case highlights the fact that patients with a history of thrombosis should be carefully evaluated for underlying MPD particularly if they show even slightly raised platelet or leukocyte counts. Early diagnosis is crucial in patients with ET as the development of thrombosis significantly affects morbidity and mortality. If diagnosed early, before the development of complications, patients can expect a good life expectancy with currently available therapy and have a low risk of future leukemic transformation or bone marrow fibrosis.

\section{Additional Information}

\section{Disclosures}

Human subjects: Consent was obtained or waived by all participants in this study. Conflicts of interest: In compliance with the ICMJE uniform disclosure form, all authors declare the following: Payment/services info: All authors have declared that no financial support was received from any organization for the submitted work. Financial relationships: All authors have declared that they have no financial relationships at present or within the previous three years with any organizations that might have an interest in the submitted work. Other relationships: All authors have declared that there are no other relationships or activities that could appear to have influenced the submitted work.

\section{References}

1. Jensen MK, de Nully Brown P, Nielsen OJ, Hasselbalch HC: Incidence, clinical features and outcome of essential thrombocythaemia in a well defined geographical area. Eur J Haematol. 2000, 65:132-139. 10.1034/j.1600-0609.2000.90236.x

2. Valla D, Casadevall N, Huisse MG, et al.: Etiology of portal vein thrombosis in adults. A prospective evaluation of primary myeloproliferative disorder. Gastroenterology. 1988, 94:1063-1069. 10.1016/00165085(88)90567-7

3. Passamonti F, Rumi E, Pungolino E, et al.: Life expectancy and prognostic factors for survival in patients with polycythemia vera and essential thrombocythemia. Am J Med. 2004, 117:755-761. 10.1016/j.amjmed.2004.06.032

4. Rosendaal FR: Venous thrombosis: a multicausal disease . Lancet. 1999, 353:1167-1173. 10.1016/S01406736(98)10266-0

5. Denninger $\mathrm{M}-\mathrm{H}$, Chait $\mathrm{Y}$, Casadevall N, et al.: Cause of portal or hepatic venous thrombosis in adults: the role of multiple concurrent factors. Hepatology. 2000, 31:587-591. 10.1002/hep.510310307

6. Singdong R, Siriboonpiputtana T, Chareonsirisuthigul T, et al.: Characterization and prognosis significance of JAK2 (V617F), MPL, and CALR mutations in Philadelphia-negative myeloproliferative neoplasms. Asian Pac J Cancer Prev. 2016, 17:4647-4653. 10.22034/apjcp.2016.17.10.4647

7. Vardiman JW, Harris NL, Brunning RD: The World Health Organization (WHO) classification of the myeloid neoplasms. Blood. 2002, 100:2292-2302. 10.1182/blood-2002-04-1199

8. Tefferi A, Vardiman JW: The diagnostic interface between histology and molecular tests in myeloproliferative disorders. Curr Opin Hematol. 2007, 14:115-122. 10.1097/MOH.0b013e32801684d9

9. Gangat N, Wolanskyj AP, Tefferi A: Abdominal vein thrombosis in essential thrombocythemia: prevalence, clinical correlates, and prognostic implications. Eur J Haematol. 2006, 77:327-333. 10.1111/j.16000609.2006.00715.x

10. Harmanci O, Bayraktar Y: Portal hypertension due to portal venous thrombosis: etiology, clinical outcomes . World J Gastroenterol. 2007, 13:2535-2540. 10.3748/wig.v13.i18.2535 